\title{
Consideraciones terapéuticas en pacientes con leucemia aguida en la era COVID-19
}

\author{
Christian O. Ramos-Peñafiel', Nidia P. Zapata-Canto², Roberta Demichelis-Gómez ${ }^{3}$, José L. Álvarez- \\ Vera4, Luara L. Arana-Luna4, José A. De La Peña-Celaya4, María E. Espitia-Ríos ${ }^{4}$ Eleazar Hernández- \\ Ruiz', Juan M. Pérez-Zúñiga ${ }^{4}$ y Martha Alvarado-Ibarra** \\ ${ }^{1}$ Clínica de Leucemia Linfoblástica Aguda, Departamento de Hematología, Hospital General de México Dr. Eduardo Liceaga; ${ }^{2}$ Clínica de Leucemias \\ Agudas, Departamento de Hematología, Instituto Nacional de Cancerología; ${ }^{3}$ Clínica de Leucemias Agudas, Departamento de Hematología, Instituto \\ Nacional de Ciencias Médicas y Nutrición Salvador Zubirán; ' ${ }^{4}$ Departamento de Hematología, Centro Médico Nacional 20 de noviembre, ISSSTE. \\ Ciudad de México, México
}

\section{Resumen}

La pandemia por enfermedad por coronavirus 2019 (COVID-19), causada por el coronavirus 2 del síndrome respiratorio agudo grave (SARS-CoV-2), ha afectado ya a 180 países. Los pacientes con cáncer/inmunosupresión a mayor edad tienen más riesgo de presentar formas graves de la enfermedad. Los pacientes con leucemia aguda son un reto para el manejo durante la pandemia. Las recomendaciones para el manejo de estos pacientes están basadas en opinión de expertos. Se trata de una población en la que hay que realizar de forma sistemática pruebas de reacción en cadena de la polimerasa para SARS-CoV-2 y diferir en la medida de lo posible la quimioterapia citotóxica en los pacientes que resulten positivos. Por otro lado, algunos de los fármacos frecuentemente utilizados como los corticosteroides, el rituximab o la asparaginasa, pueden potencialmente complicar el curso del COVID-19, por lo que se deberá de considerar diferirlos o ajustarlos en poblaciones de mayor riesgo. De la misma forma, tomando en cuenta las particularidades de cada centro, en ciertos casos se podrá considerar dar preferencia a los esquemas de tratamiento ambulatorios que nos permitan además disminuir el requerimiento transfusional. Finalmente, muchos de los pacientes con leucemia aguda son candidatos para recibir trasplante alogénico de células progenitoras hematopoyéticas (aloTCPH). Debe tomarse en cuenta la limitación de los espacios en terapia intensiva, así como el grado de inmunosupresión derivado del trasplante. La recomendación es no diferir los aloTCPH en los pacientes con una mayor riesgo de recaída de la enfermedad. Más adelante conoceremos las consecuencias de las modificaciones en el tratamiento derivadas de la pandemia sobre la leucemia.

PALABRAS CLAVE: Leucemia linfoblástica aguda. Leucemia mieloide aguda. Leucemia promielocítica aguda. COVID-19. SARS-COV-2.

\section{Therapeutic approach in acute leukemia patients in the COVID-19 era}

\begin{abstract}
The coronavirus disease 2019 (COVID-19) pandemic caused by the severe acute respiratory syndrome coronavirus 2 (SARSCoV-2) has already affected 180 countries. Older patients and patients with cancer or immunosuppression are at greater risk of severe forms of the disease. Patients with acute leukemia are challenging to manage during the pandemic. Recommendations for the management of these patients are based on expert opinion. This is a population in which polymerase chain reaction tests for SARS-CoV-2 must be performed routinely and cytotoxic chemotherapy should be deferred as far as possible in positive patients. On the other hand, some of the frequently used drugs such as corticosteroids, rituximab or asparaginase, can potentially complicate the course of COVID-19, so consideration should be given to deferring or adjusting them in higher-

Correspondencia:

*Martha Alvarado-lbarra

E-mail: normoblasto@gmail.com

DOI: 10.24875/GMM.M21000467

Gac Med Mex. 2021;157(Supl 3):S29-S34

Disponible en PubMed

www.gacetamedicademexico.com

0016-3813/C 2021 Academia Nacional de Medicina de México, A.C. Publicado por Permanyer. Este es un artículo open access bajo la licencia CC BY-NC-ND (http://creativecommons.org/licenses/by-nc-nd/4.0/).
\end{abstract}


risk populations. In the same way, considering the particularities of each center, in certain cases it may be reasonable to give preference to outpatient regimens that also allow us to decrease the transfusion requirement. Finally, many of the patients with acute leukemia are candidates to receive allogeneic hematopoietic stem cell transplantation (alloHSCT). The limitation of the spaces in intensive care units must be considered, as well as the degree of immunosuppression derived from the transplant. The recommendation is not to defer alloHSCT in patients with an increased risk of relapse. Later, we will learn about the consequences on of the modifications in treatment on leukemia derived from the pandemic.

KEY WORDS: Acute lymphoblastic leukemia. Acute myeloid leukemia. Acute promyelocytic leukemia. COVID-19. SARS-CoV-2.

\section{Introducción}

La emergente enfermedad conocida como COVID-19, causada por el coronavirus 2 del síndrome respiratorio agudo grave (SARS-CoV-2), ha cambiado la vida del mundo como lo conocemos y nos ha llevado a cambiar nuestro estilo de vida y a aprender a protegernos de una enfermedad invisible. A diferencia de la infección por coronavirus en el 2002, el cual afectó a 32 países, el nuevo SARS-CoV-2 muestra un patrón de diseminación más rápido que su antecesor, afectando a 180 países, pero con una mortalidad mucho menor $(2.09 \%$ para el SARS-CoV-2, $10.8 \%$ para el SARS-CoV del 2002 y $34.77 \%$ para el síndrome respiratorio agudo del Medio Oriente) $)^{1,2}$. Una mayor edad y la inmunosupresión se han asociado a una mayor probabilidad de enfermedad severa y fatal $(15 \%$ de mortalidad en mayores de 80 años) $)^{3}$. Muchos de los pacientes con leucemia aguda tienen estas características. En neoplasias hematológicas se ha reportado una fatalidad tan alta como del $37 \% 4$. Esto, aunado a la sobreocupación de los sistemas de salud y la necesidad de un distanciamiento social, hacen que el manejo de estos pacientes durante la pandemia sea un verdadero reto; al momento la mayor parte de las recomendaciones son extendidas por opiniones de expertos, los cuales han sugerido continuar con los esquemas terapéuticos, pero siempre bajo la consideración de una infección potencial por SARS-CoV-2.

Estas recomendaciones están inspiradas en las guías emitidas por el Programa Español de Tratamiento en Hematología (PETHEMA), la American Society of Hematology (ASH), la European Hematology Asociation (EHA), el National Institute for Health and Care Excellence (NICE) y la Organización Mundial de la Salud (OMS) 2020.

\section{Recomendaciones generales en pacientes con cáncer}

Existen algunas recomendaciones que pueden ser válidas para todo paciente con cáncer durante la pandemia por COVID-19.
- Trabajo social para atención de individuos ambulatorios:

- Crear un directorio de pacientes para establecer un sistema de comunicación a distancia que limite su desplazamiento a hospitales que atienden casos de pacientes COVID-19.

- Atención por parte de médicos locales para manejo de los síntomas generales e identificación de la sintomatología específica de riesgo.

- Implementación de telemedicina para la resolución de dudas o impartición de instrucciones.

- Pacientes ambulatorios:

- Se recomienda autoaislamiento para evitar o retrasar una infección por SARS-CoV-2, en especial en el periodo posquimioterapia.

- En caso de acudir a consulta o a toma de muestras de laboratorio, los pacientes deben de permanecer en un área segura que permita el distanciamiento.

- Diferir algunas infusiones terapéuticas (bifosfonatos, flebotomías, hierro endovenoso).

- Retrasar la administración de regímenes de quimioterapia que puedan inducir una inmunosupresión prolongada o que incrementen el riesgo de ingreso a un área de urgencias.

- Vigilar de forma estrecha a los pacientes con uso de esteroides, anticuerpos monoclonales, inhibidores de proteosoma, diferir especialmente aquellos casos que requieren dosis elevadas de esteroides.

- Administración de quimioterapia de mantenimiento:

- Valorar en cada caso el riesgo de la suspensión de terapias de mantenimiento que incluyan esteroides o anticuerpos monoclonales.

- Se recomienda continuar con los esquemas de tratamiento oral, evitando en la medida de lo posible las visitas hospitalarias.

- Administración de estrategias curativas:

- En la medida de lo posible, continuar con los esquemas con posibilidad curativa. 
- No se recomienda la presencia de cuidadores o familiares durante la hospitalización (salvo pediatría o causas de fuerza mayor).

- No se deberá utilizar presión positiva en las habitaciones de los pacientes.

- Evitar el uso de aerosoles.

- Emplear en lo posible accesos venosos que no requieran visitas frecuentes para revisión.

- Restringir el soporte hemoterápico a los casos en que esté plenamente indicado.

- A todos los pacientes con COVID-19 se les deberá realizar un electrocardiograma por riesgo de prolongación de QT.

- En aquellos casos con hipogammaglobulinemia asociada, valorar la administración de inmunoglobulina endovenosa.

- Mantener en la medida de lo posible los protocolos activos de trasplante de progenitores hematopoyéticos, valorando de manera específica el riesgo/beneficio de cada caso.

- Adecuar el timing del trasplante de progenitores hematopoyéticos o de la terapia celular a la situación de la epidemia por COVID-19 y a la realidad de cada centro, en especial en lo que respecta a disponibilidad de camas en la unidad de cuidados intensivos.

\section{Recomendaciones leucemia aguda}

Los pacientes con leucemia aguda constituyen una población con una enfermedad con comportamiento agresivo y que conlleva un riesgo de complicaciones que requieren hospitalización y apoyo transfusional. En seguida enlistamos algunos lineamientos específicos basadas en recomendaciones internacionales ${ }^{5-19}$ :

- Antes de iniciar cualquier tratamiento intensivo para la leucemia aguda, deberá asegurarse que el paciente sea COVID-19 negativo, mediante test de reacción en cadena de la polimerasa. Si se detectara infección por SARSCoV-2, no iniciar el tratamiento hasta que el paciente se recupere y la prueba de reacción en cadena de la polimerasa de COVID sea negativa (idealmente en dos muestras consecutivas separadas 48 horas).

- A todos los pacientes que ingresan por primera vez, que requieran quimioterapia intensiva, o bien aquellos de seguimiento que por la naturaleza de su enfermedad requieren hospitalización prolongada para recibir tratamiento
Tabla 1. Score de riesgoen pacientes con infección por coronavirus 2 del síndrome respiratorio agudo grave (SARS-CoV-2)

Proteína C reactiva $>150 \mathrm{mg} / \mathrm{dl}$

Dímero D mayor a $1,000 \mathrm{ng} / \mathrm{ml}$

Ferritina $>1,000 \mathrm{ng} / \mathrm{ml}$

Linfopenia $<800 / \mathrm{mm}^{3}$

Interleucina $6>40 \mathrm{pg} / \mathrm{ml}$

y además presentan evidencia de proceso infeccioso de vías respiratorias, deberán ser evaluados en forma sistemática con el score mostrado en la tabla 1.

A los pacientes con una puntuación mayor de 4 puntos se les realizará tomografía computarizada (TC) de tórax simple, además de la reacción en cadena de la polimerasa para SARS-CoV-2.

Si la TC es compatible con infección por SARSCoV-2 o la prueba de la reacción en cadena de la polimerasa es positiva, el paciente sale del área de hematología y pasa a área específica de COVID. A la resolución del cuadro y con prueba de la reacción en cadena de la polimerasa negativa, se puede valorar inicio de quimioterapia en el área de hematología.

- Tratar a los pacientes en espacios libres de COVID-19, de preferencia en cuartos aislados; deberán de ser monitorizados de forma sistemática y se deberá realizar oximetría de pulso por horario.

- Cada cuarto deberá contar con material suficiente (batas, gorros, cubrebocas, gafas de protección ocular, guantes, botas y caretas) para el personal de salud que atiende a estos pacientes.

- Todo el personal médico durante el pase de visita deberá usar el material de protección y practicará lavado de manos antes y después de cada paciente.

- El personal de enfermería deberá idealmente permanecer fijo en el servicio de hematología para la atención de este grupo de pacientes utilizando todo el material de protección ya descrito y se procurará evitar su rotación a las áreas de atención de pacientes con COVID-19. Lo mismo deberá aplicar para el personal de intendencia y camillería.

- No existe restricción para ningún fármaco de los administrados para la leucemia aguda 
linfoblástica y no linfoblástica. Se recomienda que en las consolidaciones o posremisiones se evalúe la posibilidad de reducción al $75 \%$ de la dosis de citarabina.

- Si el paciente está neutropénico, puede administrarse filgrastim (FEC-G).

- Si el paciente presenta neutropenia febril:

- Efectuar prueba de la reacción en cadena de la polimerasa para SARS-CoV-2 y radiografía de tórax, aparte de los procedimientos diagnósticos habituales de la neutropenia febril. Adicionar los estudios de la tabla 1.

- Si el paciente resulta positivo para COVID-19 deberá pasar al área COVID.

- Los enfermos que sean transferidos al área COVID serán atendidos en forma conjunta.

- Si el paciente es ambulatorio y requiere hospitalización, deberá buscarse su ingreso directo a hospitalización y evitar su estancia en admisión continua o urgencias del centro médico o de otros hospitales.

- Restringir el soporte transfusional a los casos en que esté plenamente indicado.

- Usar telemedicina siempre que sea posible. Ello es factible en los controles durante el tratamiento de mantenimiento.

- Utilizar siempre que sea posible el aislamiento a domicilio.

- Emplear en lo posible accesos venosos que no requieran visitas frecuentes para revisión.

- Adecuar el momento del trasplante de progenitores hematopoyéticos o de la terapia celular a la situación de la epidemia por COVID-19 y a la realidad de cada centro, en especial en lo que respecta a disponibilidad de camas en la unidad de cuidados intensivos.

- Si el paciente presenta infección por SARSCoV-2 durante el tratamiento con quimioterapia:

- Suspender la quimioterapia y pasar a área específica para COVID.

- Mantener el tratamiento de soporte (transfusiones, FEC-G, eltrombopag, etc.) si es necesario.

- Mantener la profilaxis o el tratamiento antiinfeccioso que el paciente requiere.

- Tratar la infección por SARS-CoV-2 con la pauta que recomienden los especialistas de enfermedades infecciosas y epidemiólogos de cada centro.

- No reiniciar la quimioterapia hasta que los síntomas hayan desaparecido y haya idealmente
Tabla 2. Recomendaciones de manejo en pacientes con leucemia linfoblástica aguda

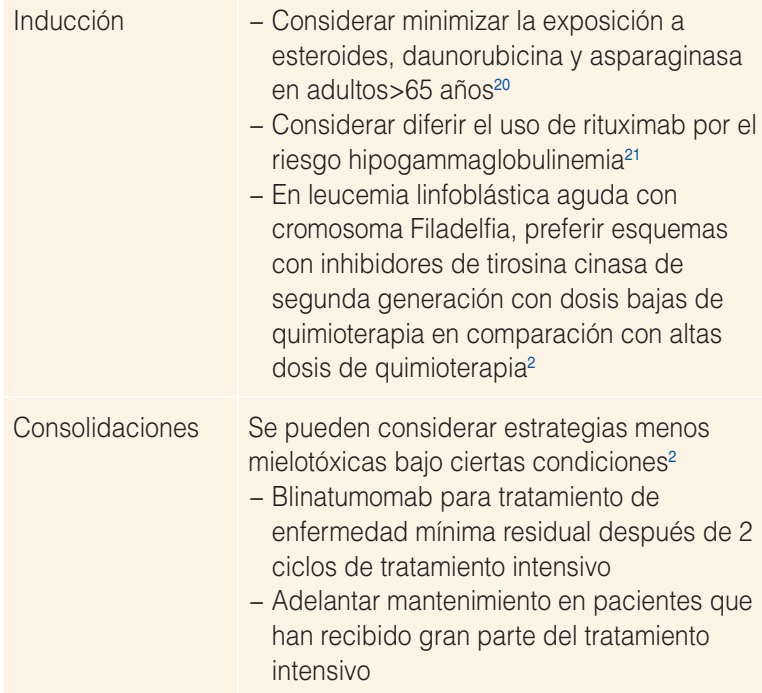

- Considerar minimizar la exposición a esteroides, daunorubicina y asparaginasa en adultos $>65$ años ${ }^{20}$

- Considerar diferir el uso de rituximab por el riesgo hipogammaglobulinemia ${ }^{21}$

- En leucemia linfoblástica aguda con cromosoma Filadelfia, preferir esquemas con inhibidores de tirosina cinasa de segunda generación con dosis bajas de quimioterapia en comparación con altas dosis de quimioterapia ${ }^{2}$

Consolidaciones Se pueden considerar estrategias menos mielotóxicas bajo ciertas condiciones ${ }^{2}$

- Blinatumomab para tratamiento de enfermedad mínima residual después de 2 ciclos de tratamiento intensivo

- Adelantar mantenimiento en pacientes que han recibido gran parte del tratamiento intensivo

Mantenimiento Considerar disminuir la dosis de esteroides y suspender vincristina en adultos mayores ${ }^{8}$

Recaída/refractario En caso de tener acceso a inmunoterapia, y trasplante se prefiere inotuzumab sobre blinatumomab para reducir la hospitalización ${ }^{8}$

En cuanto a la realización de trasplante alogénico de células progenitoras hematopoyéticas, este podrá diferirse en los pacientes en primera remisión completa. En los casos refractarios o en recaída en segunda remisión completa, se sugiere no retrasar el trasplante

dos pruebas de reacción en cadena de la polimerasa negativas para SARS-CoV-2 separadas 48 horas.

- Retrasar en lo posible la administración de l-asparaginasa en caso de leucemia linfoblástica aguda, tras la resolución de la infección, por el mayor riesgo trombótico asociado a la COVID-19.

- Notificar el caso a las autoridades correspondientes.

- En la atención y seguimiento de pacientes en la consulta externa solo se permitirá un acompañante cuando el paciente no pueda valerse por sí mismo, en el resto de los casos solo entrará el paciente y en ambos casos se deberá mantener la sana distancia.

- En caso de requerir revisión del enfermo en la consulta externa, el médico deberá utilizar equipo de protección correspondiente (cubrebocas, goggles, bata y guantes).

- En las valoraciones de pacientes hematológicos en admisión continua o urgencias el personal de 
Tabla 3. Recomendaciones de manejo en pacientes con leucemia mieloide aguda

Inducción

Consolidaciones

$$
\begin{aligned}
& \text { Los pacientes que sean candidatos } \\
& \text { a consolidación con dosis altas de } \\
& \text { citarabina, se pueden dar } 3 \text { en lugar de } 4 \\
& \text { consolidaciones y la dosis de } 1.5 \mathrm{~g} / \mathrm{m}^{2} \text { en } \\
& \text { lugar de } 3 \mathrm{~g} / \mathrm{m}^{2} 8 \\
& \text { El de trasplante alogénico de células } \\
& \text { progenitoras hematopoyéticas en pacientes } \\
& \text { que no sean de alto riesgo y que tengan } \\
& \text { enfermedad mínima residual negativa, podrá } \\
& \text { ser diferido } \\
& \text { A medida de lo posible, se preferirá el } \\
& \text { tratamiento basado en terapias blanco } \\
& \text { con gilteritinib e inhibidores de la } \\
& \text { isocitrato-deshidrogenasa } 1 \text { y } 2
\end{aligned}
$$

Recaída/refractario A medida de lo posible, se preferirá el y trasplante

salud deberá usar el equipo de protección anteriormente descrito hasta que se hayan descartado objetivamente factores de riesgo para infección por SARS-CoV-2.

- Como norma general, mantener un contacto estrecho con los equipos que encabezan COVID para adaptarse a las circunstancias cambiantes de la epidemia y a los nuevos conocimientos que se van adquiriendo de esta infección.

- Todos los pacientes antes de recibir su tratamiento deberán firmar su carta de consentimiento informado.

\section{Recomendaciones específicas por tipo de leucemia}

En las tablas 2 y 3 podemos ver las recomendaciones específicas por tipo de leucemia que se suman a las recomendaciones generales.

\section{Conclusiones}

La pandemia por COVID-19 modificó de forma sustancial el manejo de la medicina. La leucemia aguda no son la excepción, al ser una población susceptible y que requiere de una gran infraestructura médica y hospitalaria. Las recomendaciones están enfocadas en disminuir el riesgo de infección y complicaciones por SARS-CoV-2 tratando de sacrificar lo menos posible el pronóstico de la enfermedad de base. Algunas estrategias, como implementar telemedicina para el seguimiento de nuestros pacientes, serán positivas. Sin embargo, será más adelante cuando podamos ver el daño colateral que dejará esta pandemia en los pacientes con neoplasias hematológicas.

\section{Conflicto de intereses}

Los autores declaran no tener conflicto intereses.

\section{Financiamiento}

La presente investigación no ha recibido ninguna beca específica de agencias de los sectores público, comercial, o sin ánimo de lucro.

\section{Responsabilidades éticas}

Protección de personas y animales. Los autores declaran que para esta investigación no se han realizado experimentos en seres humanos ni en animales.

Confidencialidad de los datos. Los autores declaran que en este artículo no aparecen datos de pacientes.

Derecho a la privacidad y consentimiento informado. Los autores declaran que en este artículo no aparecen datos de pacientes.

\section{Bibliografía}

1. Meo SA, Alhowikan AM, Al-Khlaiwi T, Meo IM, Halepoto DM, Iqbal M, et al. Novel coronavirus 2019-nCoV: prevalence, biological and clinical characteristics comparison with SARS-CoV and MERS-CoV. Eur Rev Med Pharmacol Sci. 2020;24(4):2012-19.

2. Zeidan AM, Boddu OC, Patnaik MM, Bewersdorf JP, Stahl M, Rampal RK, et al. Special considerations in the management of adult patients with acute leukaemias and myeloid neoplasms in the COVID-19 era: recommendations from a panel of international experts. Lancet Haematol. 2020;7(8):e601-e612.

3. Wu Z, McGoogan JM. Characteristics of and important lessons from the coronavirus disease 2019 (COVID-19) outbreak in China: summary of a report of 72314 cases from the Chinese Center for Disease Control and Prevention. JAMA. 2020;323:1239-42 .

4. Mehta V, Goel S, Kabarriti R, Cole D, Goldfinger M, Acuna-Villaorduna A, et al. Case fatality rate of cancer patients with COVID-19 in a New York hospital system. Cancer Discov. 2020;10(7):935-41. 
5. Guan WJ, Ni ZY, Hu Y, Liang WH, Ou CQ, He JX, et al. Clinical characteristics of coronavirus disease 2019 in China. N Engl J Med. 2020;382(18):1708-20.

6. Liang W, GuanW, Chen R, Wan W, Li J, Xu K, et al. Cancer patients in SARS-CoV-2 infection: a nationalwide análisis in China. Lancet Oncol. 2020;21:335-7.

7. Jazieh AR, Alenazi TH, Alhejazi A, Al Safi F, Al Olayan A. Outcome of oncology patients infected with coronavirus. JCO Global Oncol. 2020:4:471-5.

8. Stock W, Patel A, O'Dwyer K, Bassan R, Zhou X, Huang WJ, et al. COVID-19 and adult ALL: Frequently asked questions [Internet]. American Society of Hematology [última actualización: 22/01/2021]. Disponible en: https://www.hematology.org/covid-19/covid-19-and-all

9. Tallman M, Rollig C, Zappasodi P, Schiller G, Mannis G, Olin R, et al. COVID-19 and acute myeloid leukemia: Frequently asked questions [Internet]. American Society of Hematology [última actualización: 22/01/2021]. Disponible en: https://www.hematology.org/covid-19/covid-19-and-acute-myeloid-leukemia

10. NCCN Guidelines ${ }^{\circledR}$ \& Clinical Resources. NCCN Guidelines. Version 1 2020 [Internet]. Plymouth Meeting, PA: National Comprehensive Cancer Network. Disponible en: https://www.nccn.org/professionals/physician_ gls/default_nojava.aspx

11. Lipsitch M, Swerdlow DL, Finelli L. Defining the epidemology of COVID-19 studies needed. N Engl J Med. 2020;382:1194-6.

12. Zhang J, Zhou L, Yang Y, Peng W, Wang W, Chen X. Therapeutic and triage strategies for 2019 novel coronavirus disease in fever clinics. Lancet Respir Med. 2020;8(3):e11-e12.

13. Maxmen A. More tan 80 clinical trials launch to test coronavirus treatments. Nature. 2020;578(7795):347-8.

14. Zumla A, Hui DS, Azhar El, Memish ZA, Maeurer M. Reducing mortality from 2019-nCoV: host directed therapies should be an option. Lancet. 2020;395(10224):E35-E36

15. Morse J, Lalonde T, Xu S, Liu WR. Learning from the past: Possible urgent prevention and treatment options for severe acute respiratory infections caused by 2019-nCoV. Chembiochem. 2020;21(5):730-8.
16. World Health Organization. Clinical management of severe acute respiratory infection when novel coronavirus (2019-nCoV) infection is suspected: Interim guidence, 13 March 2020 [Internet]. World Health Organization [consultado: 09/04/2020]. Disponible en: https://apps.who.int/iris/ handle $/ 10665 / 331446$ ? search-result=true\&query=10665\%2F331446\&scope $=\&$ rpp $=10 \&$ sort_by=score\&order $=$ desc

17. World Health Organization. Coronavirus disease (COVID-19) Weekly Epidemiological Update and Weekly Operational Update [Internet]. World Health Organization [consultado: 09/04/2020]. Disponible en: https://www. who.int/emergencies/diseases/novel-coronavirus-2019/situation-reports/

18. Center for Disease Control and Prevention. COVID Data Tracker [Internet]. Center for Disease Control and Prevention [consultado: 09/04/2020]. Disponible en: https://covid.cdc.gov/covid-data-tracker/?CDC_AA_ref$\mathrm{Val}=\mathrm{https} \% 3 \mathrm{~A} \% 2 \mathrm{~F} \% 2 \mathrm{Fwww}$.cdc.gov\%2Fcoronavirus\%2F2019-ncov\%2Fcases-updates\%2Fcases-in-us.html\#cases casesper100klast7days

19. Kollias A, Kiriakoulis K, Dimakakos E, Poulakou G, Stergiou GS, Syrigos K. Thromboembolic risk and anticoagulant therapy in COVID-19 patients: Emerging evidence and call for action. Br J Haematol 2020. 2020;189(5):846-7.

20. Lee N, Allen Chan KC, Hui DS, Ng EK, Wu A, Chiu RW, et al. Effects of early corticosteroid treatment on plasma SARS-associated coronavirus RNA concentrations in adult patients. J Clin Virol. 2004:31:304-9.

21. Casulo C, Maragulia J, Zelenetz AD. Incidence of hipogammaglobulinemia in patients receiving Rituximab and the use of intravenous immunoglobulin for recurrent infections. Clin Lymphoma Myeloma Leuk. 2013;13(2):106-11.

22. The National Cancer Research Institute Acute Myeloid Leukaemia Working Party. Recommendations for the management of patients with AML during the COVID19 outbreak: a statement from the NCRI AML Working Party [Internet]. National Cancer Research Institute Acute Myeloid Leukaemia Working Party [última actualización: 23/03/2020]. Disponible en: https:// www.rcpath.org/uploads/assets/d23030b6-7379-4f80-9ed9178c5f864343/ Recommendations-for-the-management-of-patients-with-acute-myeloid-leukaemia-AML-during-the-COVID19-outbreak.pdf 